\title{
Combining Diffusion and Grey Models Based on Evolutionary Optimization Algorithms to Forecast Motherboard Shipments
}

\author{
Fu-Kwun Wang, ${ }^{1,2}$ Yu-Yao Hsiao, ${ }^{2}$ and Ku-Kuang Chang ${ }^{3}$ \\ ${ }^{1}$ Department of Industrial Management, National Taiwan University of Science and Technology, \\ Taipei 106, Taiwan \\ ${ }^{2}$ Graduate Institute of Management, National Taiwan University of Science and Technology, \\ Taipei 106, Taiwan \\ ${ }^{3}$ Department of Logistic Management, Takming University of Science and Technology, Taipei 114, Taiwan
}

Correspondence should be addressed to Fu-Kwun Wang, fukwun@mail.ntust.edu.tw

Received 21 February 2012; Revised 12 June 2012; Accepted 15 June 2012

Academic Editor: Yi-Chung Hu

Copyright (c) 2012 Fu-Kwun Wang et al. This is an open access article distributed under the Creative Commons Attribution License, which permits unrestricted use, distribution, and reproduction in any medium, provided the original work is properly cited.

It is important for executives to predict the future trends. Otherwise, their companies cannot make profitable decisions and investments. The Bass diffusion model can describe the empirical adoption curve for new products and technological innovations. The Grey model provides shortterm forecasts using four data points. This study develops a combined model based on the rolling Grey model (RGM) and the Bass diffusion model to forecast motherboard shipments. In addition, we investigate evolutionary optimization algorithms to determine the optimal parameters. Our results indicate that the combined model using a hybrid algorithm outperforms other methods for the fitting and forecasting processes in terms of mean absolute percentage error.

\section{Introduction}

Taiwanese motherboard manufacturers create $98.5 \%$ of the worldwide desktop motherboards and dominate the global desktop motherboard market [1]. However, this industry's growth rate has slowed because of the trend of replacing desktop PCs with notebooks or netbooks. In addition, the aggressive pricing by notebook/netbook manufacturers has diminished desktop motherboard sales. It is important to develop a new forecasting model for this rapidly changing market and to compare its results with other forecasting models. These results can assist manufactures in making decisions on future expansion and investment.

Several studies have proposed time-series models for industrial production, demonstrating the applicability of time-series models to industrial production forecasting. These 
models typically require large amounts of data. However, Hsu [2] proved that the Grey model (GM), developed by Deng [3], requires minimal data and is the best model for limited data prediction. Furthermore, GM can forecast in a completive environment where decision makers have limited historical data. Chang et al. [4] applied a variable $p$ value to a rolling Grey model (RGM) to forecast semiconductor production in Taiwan. Akay and Atak [5] used the Grey prediction model with a rolling mechanism to forecast electricity demand in Turkey. Hsu and Wang [6,7] used the Bayesian method to improve $\operatorname{GM}(1,1)$ for forecasting the integrated circuit industry.

The Bass diffusion model [8] has been used to develop product life cycle curves and to forecast the sales of the initial purchases of new products. Tseng and $\mathrm{Hu}$ [9] combined fuzzy regression with the Bass model to develop a quadratic interval Bass diffusion model. Tsaur [10] used the fuzzy grey regression model to predict the liquid critical display (LCD) television market. Based on empirical data analysis, fuzzy grey regression is capable of accurate forecasting and can give decision makers various scenarios. $\mathrm{Wu}$ and $\mathrm{Chu}$ [11] used Gompertz, Logistic, Bass, and time-series autoregressive moving average (ARMA) models to forecast mobile telephone subscription in Taiwan. Hsiao and Wang [12] applied the $\operatorname{GM}(1,1)$, $\operatorname{RGM}(1,1)$, and Bass diffusion models to predict trends in the global copper clad laminate industry.

This study proposes a combined model based on the rolling Grey and Bass diffusion models to forecast the sale of Taiwanese motherboards more accurately. This study is organized as follows: Section 2 reviews the Bass diffusion model and the RGM(1,1); Section 3 introduces a combined model featuring an evolutionary optimization algorithm; in Section 4 we use the combined model to forecast motherboard shipments, comparing its results with those of the RGM(1,1) and Bass diffusion models; finally, we provide a conclusion.

\section{Bass Diffusion Model and RGM(1,1)}

This section presents a discussion on two forecasting models the Bass diffusion and $\operatorname{RGM}(1,1)$ models. The Bass diffusion model [13] is given as

$$
n(t)=m \times[F(t)-F(t-1)]+\varepsilon,
$$

where $F(t)=\left[1-e^{-(p+q) t}\right] /\left[1+(q / p) \times e^{-(p+q) t}\right], n(t)=$ sales at time $t, m$ is the number of eventual adopters, $F(t)$ is the cumulative distribution of adoptions at time $t, p$ is the coefficient of innovation, $q$ is the coefficient of imitation, and $\varepsilon$ is the normally distributed random error term with mean zero and variance $\sigma^{2}$. The adopter's probability density function $f(t)$ for adoption at time $t$ is given by

$$
f(t)=\frac{\left[\left((p+q)^{2} / p\right) \times e^{-(p+q) t}\right]}{\left[1+(q / p) \times e^{-(p+q) t}\right]^{2}} .
$$

Bass [13] used the ordinary least squares (OLS) method to estimate the parameters. Schmittlein and Mahajan [14] used the maximum likelihood estimation (MLE) method to improve the estimation. Srinivsan and Mason [15] used a nonlinear least square estimation (NLS) method to obtain the valid error estimates. Nonlinear models are more difficult to fit 
than linear models. Venkatesan and Kumar [16] used genetic algorithms (GAs) to estimate the parameters, and these were consistent with the NLS method.

Grey theory is used for systems that have uncertain and imperfect information [3]. It requires only four data points to construct a prediction model. Grey prediction has three basic operations: accumulated generating operator (AGO), the inverse accumulating operator (IAGO), and the GM. The steps of the $\operatorname{RGM}(1,1)$ model are given as follows.

Step 1. Original time sequence with $n$ samples is expressed as

$$
X^{(0)}=\left[x^{(0)}(1), x^{(0)}(2), x^{(0)}(3), \ldots, x^{(0)}(n)\right]
$$

An AGO operator is used to convert the original series into monotonically increasing series:

$$
X^{(1)}=\left[x^{(1)}(1), x^{(1)}(2), x^{(1)}(3), \ldots, x^{(1)}(n)\right]
$$

where $x^{(1)}(k)=\sum_{i=1}^{k} x^{(0)}(i)$.

Step 2. The first-order differential equation for the GM $(1,1)$ model is given by

$$
\frac{d X^{(1)}}{d t}+a \times X^{(1)}=b,
$$

where $t$ denotes the independent variables in the system, $a$ represents the developed coefficient, and $b$ is the Grey controlled variable. The parameters of $a$ and $b$ can be obtained using the OLS method. Thus, we have

$$
\widehat{u}=\left[\begin{array}{l}
a \\
b
\end{array}\right]=\left(B^{T} B\right)^{-1} \times B^{T} \times Y_{N}
$$

where the accumulated matrix

$$
B=\left[\begin{array}{cc}
-\left[P_{x}^{(1)}(i)+(1-P) x^{(1)}(i+1)\right] & 1 \\
-\left[P_{x}^{(1)}(i+1)+(1-P) x^{(1)}(i+2)\right] & 1 \\
\vdots & \vdots \\
-\left[P_{x}^{(1)}(k-1)+(1-P) x^{(1)}(k)\right] & 1
\end{array}\right],
$$

$P$ is equal 0.5 in the original model, and $Y_{N}=\left[x^{(0)}(i+1), x^{(0)}(i+2), \ldots, x^{(0)}(k)\right]^{T}$.

Step 3. The approximate relationship can be obtained by substituting $\hat{u}$ (determined in the differential equation in Step 2) as follows:

$$
\widehat{x}^{(1)}(t+1)=\left[x^{(0)}(1)-\frac{b}{a}\right] \times e^{-a t}+\frac{b}{a} .
$$




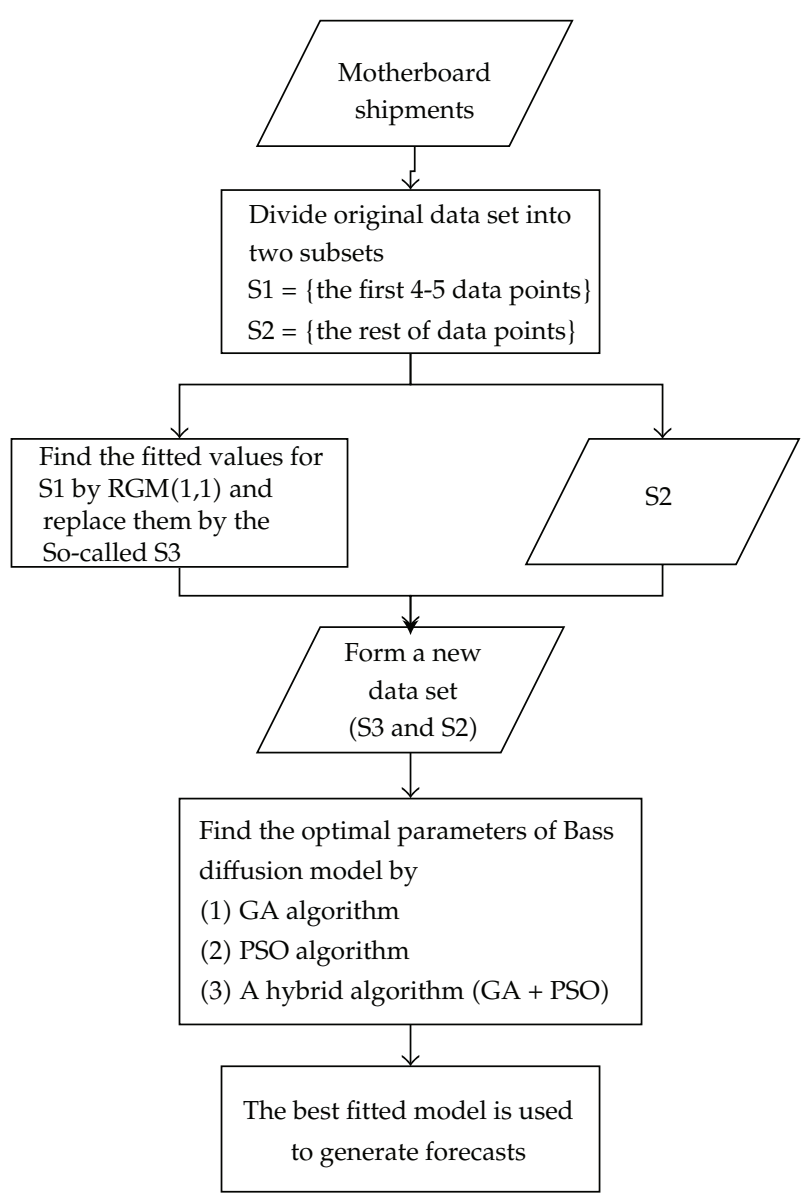

Figure 1: Procedures of the proposed method.

Supposing that $\widehat{x}^{(1)}(1)=\widehat{x}^{(0)}(1)$, the sequence one-order IAGO is acquired. Thereafter, the sequence can be obtained as $\widehat{x}^{(0)}(t+1)=\widehat{x}^{(1)}(t+1)-\widehat{x}^{(1)}(t)$.

Given $t=1,2, \ldots, k$, the sequence of reduction is as follows:

$$
\widehat{X}^{(0)}(i ; k)=\left(\widehat{x}^{(0)}(1), \widehat{x}^{(0)}(2), \ldots, \widehat{x}^{(0)}(k+1)\right),
$$

where $\widehat{x}^{(0)}(k+1)$ is the Grey elementary forecasting value for $\widehat{x}^{(0)}(k+1)$.

\section{Combined Model}

Combining forecasts minimizes errors [18], and many studies have demonstrated their value [19-21]. In this section, we present a combined model based on the Bass diffusion model and the $\operatorname{RGM}(1,1)$ (see Figure 1). The three major steps of the proposed combined model are as follows. 
Step 1. A new data set should be formed. The $\operatorname{RGM}(1,1)$ usually provides better fitted values for early periods than does the Bass diffusion model. Next, the original data points are replaced by some predicted values by the $\operatorname{RGM}(1,1)$ to form a new data set.

Step 2. The optimal parameters of the Bass diffusion model should be found for the original data and a new data set. A hybrid algorithm based on the GA with PSO has been successfully applied to real-world engineering design problems [22, 23]. This study used a hybrid algorithm that couples the GA with PSO to optimize the estimates of the parameters for the Bass diffusion model. We then investigated three algorithms to obtain the parameters of the Bass diffusion model. First, we used a nonlinear algorithm to obtain the initialized estimates of the model. In addition, the confidence intervals for the parameters were used to determine the range of the parameters for PSO and the GA. The descriptions of these three algorithms are as follows.

(1) GA: the estimated parameters can be obtained easily using Evolver Software [24]. The minimized function is defined as MAPE. Here, the population size is set by 50 , and the crossover rate and the mutation rate are set as 0.6 and 0.2 , respectively.

(2) PSO algorithm: we used PSO operators (velocity and position updates) to update the individual with the worst fitness. Clerc and Kennedy [25] created constriction factor $k$, improving the ability of PSO to constrain and control velocities. These equations are given by

$$
\begin{gathered}
V_{\mathrm{id}}^{\mathrm{New}}=k \times V_{\mathrm{id}}^{\mathrm{old}}+\left[c_{1} \times r_{1} \times\left(p_{\mathrm{id}}-x_{\mathrm{id}}^{\mathrm{old}}\right)+c_{2} \times r_{2} \times\left(p_{\mathrm{gd}}-x_{\mathrm{id}}^{\mathrm{old}}\right)\right], \\
x_{\mathrm{id}}^{\mathrm{New}}=x_{\mathrm{id}}^{\text {old }}+V_{\mathrm{id}}^{\mathrm{New}},
\end{gathered}
$$

where $k$ is an inertia weight, $c_{1}$ and $c_{2}$ are two positive constants called acceleration coefficients, and $r_{1}$ and $r_{2}$ are random, uniformly distributed numbers [0,1]. The inertia weight can be obtained by $k=2 /\left|2-c-\sqrt{c^{2}-4 c}\right|$, where $c=c_{1}+c_{2}>4$. For example, if $c=4.1$, then $w=0.729$. As $c$ increases above $4.0, k$ becomes smaller. The input parameters are particle size $=20$, max iterations $=100, w=0.729$, and $c_{1}=c_{2}=2.05$.

(3) A hybrid algorithm: this hybrid algorithm couples the GA with PSO. First, we used the PSO algorithm to obtain the estimated parameters. Thereafter, the estimated parameters were improved using the GA.

Step 3. The fitted Bass diffusion model can be used to generate forecast values.

A common measure of examining the forecasting ability of a model is defined as MAPE $=\sum_{t=1}^{n}\left(\left|\left(A_{t}-F_{t}\right) / A_{t}\right|\right) / n$, where $A_{t}$ is actual value in period $t, F_{t}$ is forecast value in period $t$, and $n$ is the number of periods used in the calculation. A low MAPE value shows an excellent forecasting ability (Table 1).

\section{Application in Motherboard Shipments}

The motherboard shipment data from 1998 to 2010 are shown in Table 2. In this study we assumed that the historical data from 1998 to 2009 are known. The holdout period is the 
Table 1: Criteria for forecasting accuracy [17].

\begin{tabular}{lc}
\hline MAPE (\%) & Forecasting power \\
\hline$<10$ & Excellent \\
$10-20$ & Good \\
$20-50$ & Reasonable \\
$>50$ & Incorrect \\
\hline
\end{tabular}

Table 2: Motherboard shipment data [1].

\begin{tabular}{lc}
\hline Year & Shipment (unit $=$ thousand) \\
\hline 1998 & 54371 \\
1999 & 64378 \\
2000 & 84372 \\
2001 & 80565 \\
2002 & 86554 \\
2003 & 103509 \\
2004 & 107987 \\
2005 & 113354 \\
2006 & 111117 \\
2007 & 109097 \\
2008 & 96743 \\
2009 & 84374 \\
2010 & 68687 \\
\hline
\end{tabular}

year 2010. First, the estimated parameters of the Bass diffusion model were obtained using the GA. Second, the rolling interval for the $\operatorname{RGM}(1,1)$ was set to five. Thereafter, we obtained eight parameter settings from 1998 to 2009. Finally, the estimated parameters of the combined model were obtained using three evolutionary algorithms (GA, PSO, and a hybrid). All five methods were used to compare the fitted values for the historical periods and the 1-yearahead forecast.

The parameter values of all five methods are shown in Table 3, and their forecasting results are listed in Table 4. The MAPE values of these five methods from 1998 to 2009 are $3.692 \%$ for the Bass diffusion model using the GA, 3.395\% for the $\operatorname{RGM}(1,1), 2.736 \%$ for the combined model using the GA, $2.480 \%$ for the combined model using PSO, and $2.476 \%$ for the combined model using a hybrid algorithm. For the 1-year-ahead forecast for 2010, the MAPE values of the five methods were $10.96 \%$ for the Bass diffusion model using the GA, $15.94 \%$ for the $\operatorname{RGM}(1,1), 9.06 \%$ for the combined model using the GA, $4.71 \%$ for the combined model using PSO, and $4.69 \%$ for the combined model using a hybrid algorithm. Our results demonstrate that the proposed combined model using a hybrid algorithm outperformed the other methods for the fitting and forecasting processes in terms of MAPE, as shown in Figure 2. 
Table 3: Parameter values of all methods.

\begin{tabular}{lll}
\hline Year & Method & Parameters \\
\hline $1998-2009$ & Bass diffusion model with GA & $m=1512404 ; p=0.0321 ; q=0.2255$ \\
\hline $1998-2002$ & & $a=-0.0776 ; b=63299.8$ \\
$1999-2003$ & & $a=-0.0748 ; b=71247.8$ \\
$2000-2004$ & & $a=-0.1042 ; b=67427.2$ \\
$2001-2005$ & RGM(1,1) & $a=-0.0806 ; b=80632.2$ \\
$2002-2006$ & & $a=-0.0255 ; b=101302.4$ \\
$2003-2007$ & & $a=-0.0010 ; b=110072.1$ \\
$2004-2008$ & & $a=0.0473 ; b=123165.0$ \\
$2005-2009$ & Combined model with GA & $a=0.0902 ; b=129711.4$ \\
\hline $2003-2009$ & Combined model with PSO & $m=1483972 ; p=0.0311 ; q=0.2369$ \\
$2003-2009$ & Combined model with hybrid & $m=1419290 ; p=0.0288 ; q=0.2594$ \\
$2003-2009$ & & $m=1420173 ; p=0.0289 ; q=0.2589$ \\
\hline
\end{tabular}

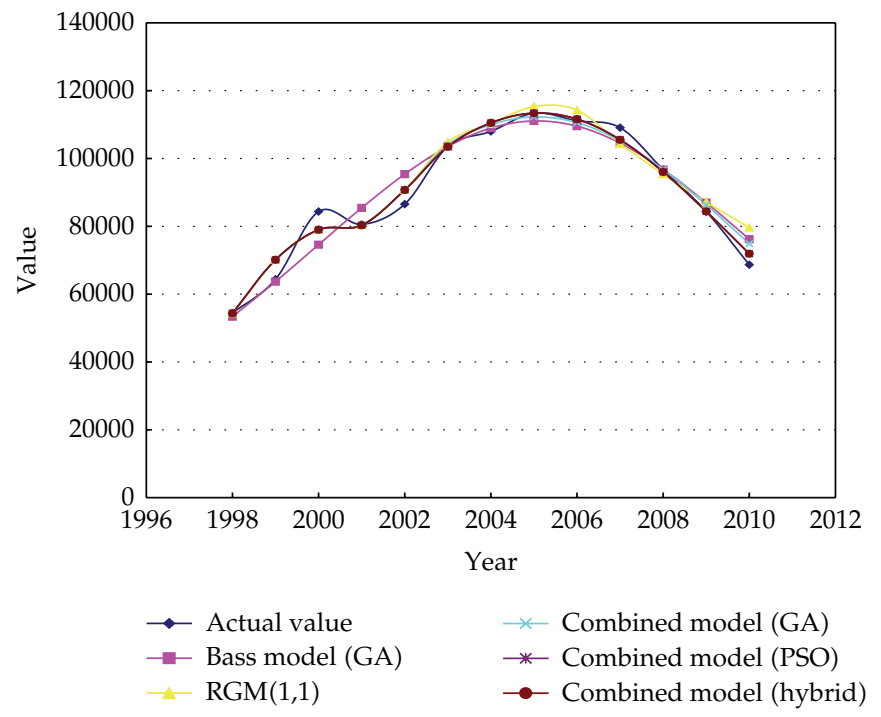

Figure 2: Forecasting results for all models.

\section{Conclusions}

This study presented a combined model that combined the Bass diffusion model with the $\operatorname{RGM}(1,1)$ to forecast motherboard shipments. In addition, we investigated evolutionary optimization algorithms to determine the optimal parameters. The results indicate that the combined model using a hybrid algorithm provides excellent MAPE improvement. We conclude that the combined model, using a hybrid algorithm, is suitable for forecasting in the motherboard industry.

Future research will include a modeling comparison of our model and the Support Vector Machine (SVM) model using a DNA optimization method [26]. 


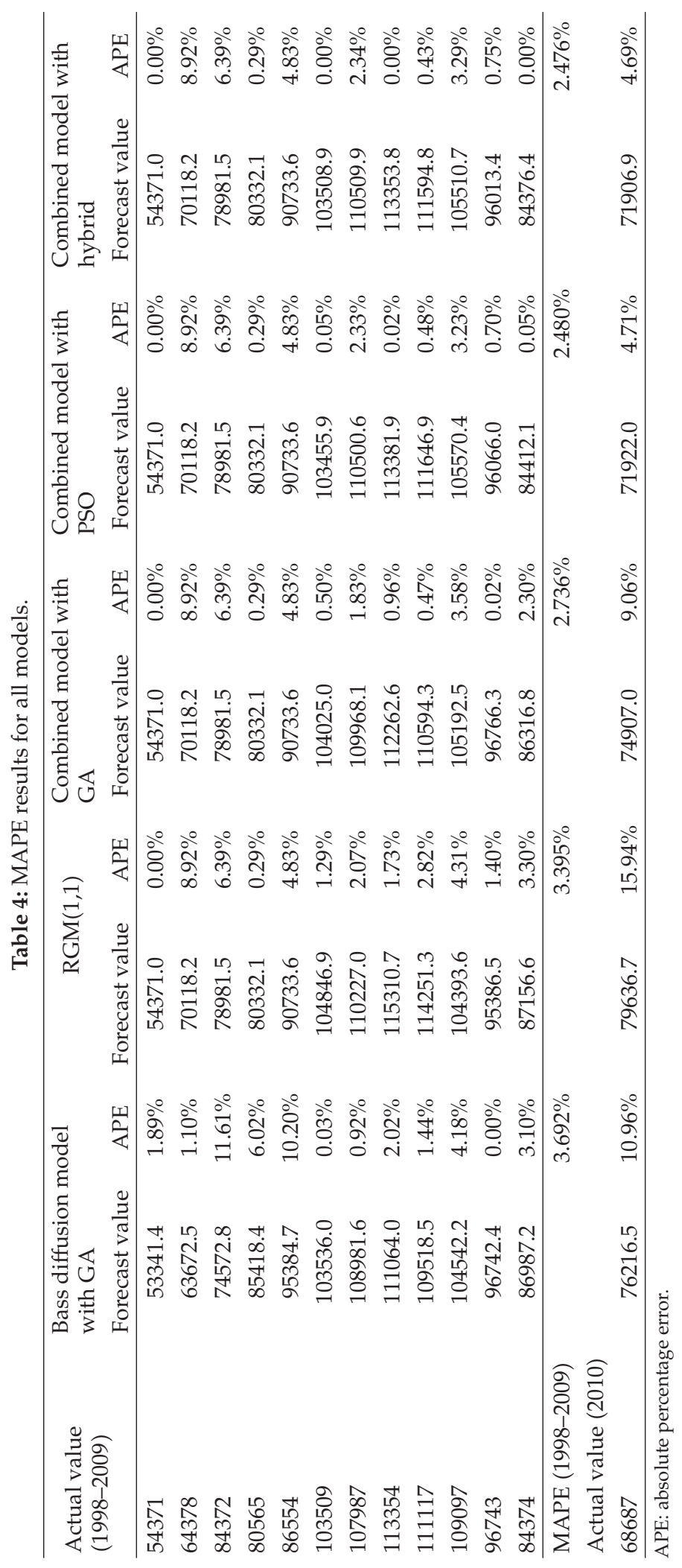




\section{Acknowledgments}

The authors gratefully acknowledge the referees of this paper who helped clarify and improve this paper. They are also thankful for the financial support from NSC in Taiwan.

\section{References}

[1] Market Intelligence Center (MIC), Taiwan Motherboard Statistics Data, MIC, Taipei, Taiwan.

[2] L. C. Hsu, "Applying the grey prediction model to the global integrated circuit industry," Technological Forecasting and Social Change, vol. 70, no. 6, pp. 563-574, 2003.

[3] J. L. Deng, “Introduction to grey system theory,” Journal of Grey System, vol. 1, no. 1, pp. 1-24, 1989.

[4] S. C. Chang, H. C. Lai, and H. C. Yu, "A variable P value rolling grey forecasting model for Taiwan semiconductor industry production," Technological Forecasting and Social Change, vol. 72, no. 5, pp. 623-640, 2005.

[5] D. Akay and M. Atak, "Grey prediction with rolling mechanism for electricity demand forecasting of Turkey," Energy, vol. 32, no. 9, pp. 1670-1675, 2007.

[6] L. C. Hsu and C. H. Wang, "Forecasting the output of integrated circuit industry using a grey model improved by the Bayesian analysis," Technological Forecasting and Social Change, vol. 74, no. 6, pp. 843853, 2007.

[7] L. C. Hsu and C. H. Wang, "Forecasting integrated circuit output using multivariate grey model and grey relational analysis," Expert Systems with Applications, vol. 36, no. 2, pp. 1403-1409, 2009.

[8] F. M. Bass, "Comments on: a new product growth for model consumer durables," Management Science, vol. 50, no. 12, pp. 1833-1840, 2004.

[9] F. M. Tseng and Y. C. Hu, "Quadratic-interval Bass model for new product sales diffusion," Expert Systems with Applications, vol. 36, no. 4, pp. 8496-8502, 2009.

[10] R. C. Tsaur, "Forecasting analysis by using fuzzy grey regression model for solving limited time series data," Soft Computing, vol. 12, no. 11, pp. 1105-1113, 2008.

[11] F. S. Wu and W. L. Chu, "Diffusion models of mobile telephony," Journal of Business Research, vol. 63, no. 5, pp. 497-501, 2010.

[12] Y. Y. Hsiao and F. K. Wang, "Forecasting analysis for global copper clad laminate market," Journal of Convergence Information Technology, vol. 7, no. 3, pp. 250-258, 2012.

[13] F. M. Bass, "A new product growth for model consumer durables," Management Science, vol. 50, no. 12, pp. 1825-1832, 2004.

[14] D. C. Schmittlein and V. Mahajan, "Maximum likelihood estimation for an innovation diffusion model of new product acceptance," Marketing Science, vol. 1, pp. 57-78, 1982.

[15] V. Srinivasan and C. H. Mason, "Nonlinear least squares estimation of new product diffusion models," Marketing Science, vol. 5, pp. 169-178, 1986.

[16] R. Venkatesan and V. Kumar, "A genetic algorithms approach to growth phase forecasting of wireless subscribers," International Journal of Forecasting, vol. 18, no. 4, pp. 625-646, 2002.

[17] C. D. Lewis, Industrial and Business Forecasting Methods, Butterworth Scientific, London, UK, 1982.

[18] J. S. Armstrong, "Combining forecasts: the end of the beginning or the beginning of the end?" International Journal of Forecasting, vol. 5, no. 4, pp. 585-588, 1989.

[19] D. W. Bunn, “Combining forecasts," European Journal of Operational Research, vol. 33, no. 3, pp. 223-229, 1988.

[20] F. K. Wang and K. K. Chang, "Adaptive neuro-fuzzy inference system for combined forecasts in a panel manufacturer," Expert Systems with Applications, vol. 37, no. 12, pp. 8119-8126, 2010.

[21] C. Christodoulos, C. Michalakelis, and D. Varoutas, "On the combination of exponential smoothing and diffusion forecasts: an application to broadband diffusion in the OECD area," Technological Forecasting and Social Change, vol. 78, no. 1, pp. 163-170, 2011.

[22] C. F. Juang, "A hybrid of genetic algorithm and particle swarm optimization for recurrent network design," IEEE Transactions on Systems, Man, and Cybernetics B, vol. 34, no. 2, pp. 997-1006, 2004.

[23] S. Jeong, S. Obayashi, and Y. Minemura, "Application of hybrid evolutionary algorithms to low exhaust emission diesel engine design," Engineering Optimization, vol. 40, no. 1, pp. 1-16, 2008.

[24] Evolver Software, Version 4.0.2, Palisade Corporation, Newfield, NY, USA, 2000. 
[25] M. Clerc and J. Kennedy, "The particle swarm-explosion, stability, and convergence in a multidimensional complex space," IEEE Transactions on Evolutionary Computation, vol. 6, no. 1, pp. 58-73, 2002.

[26] X. H. Yang and Y. Q. Li, "DNA optimization threshold autoregressive prediction model and its application in ice condition time series," Mathematical Problems in Engineering, vol. 2012, pp. 1-10, 2012. 


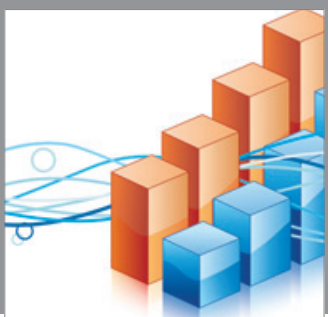

Advances in

Operations Research

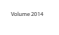

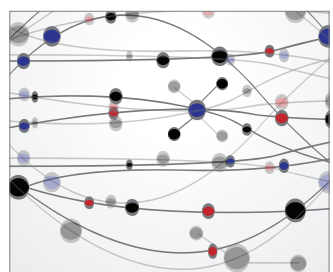

\section{The Scientific} World Journal
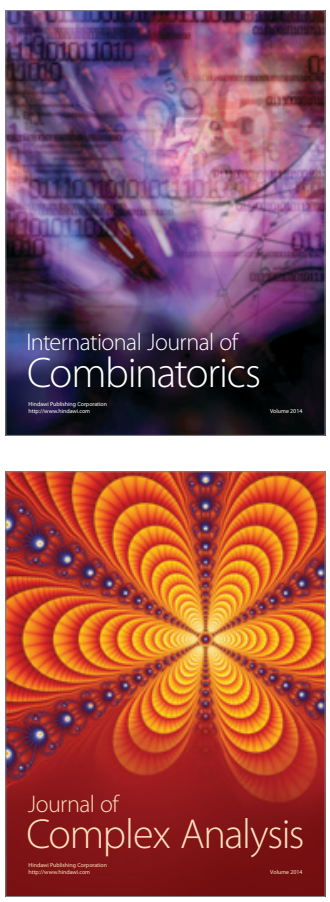

International Journal of

Mathematics and

Mathematical

Sciences
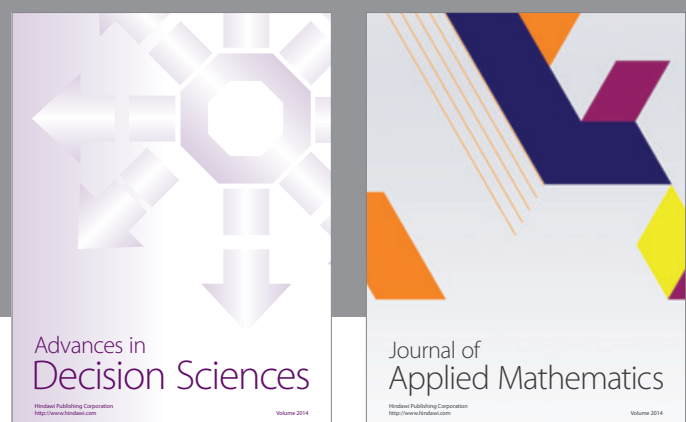

Journal of

Applied Mathematics
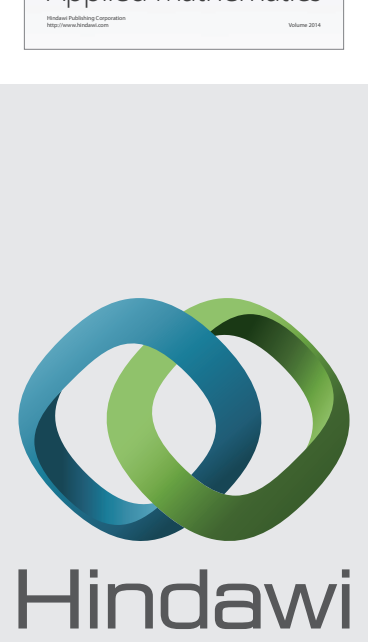

Submit your manuscripts at http://www.hindawi.com
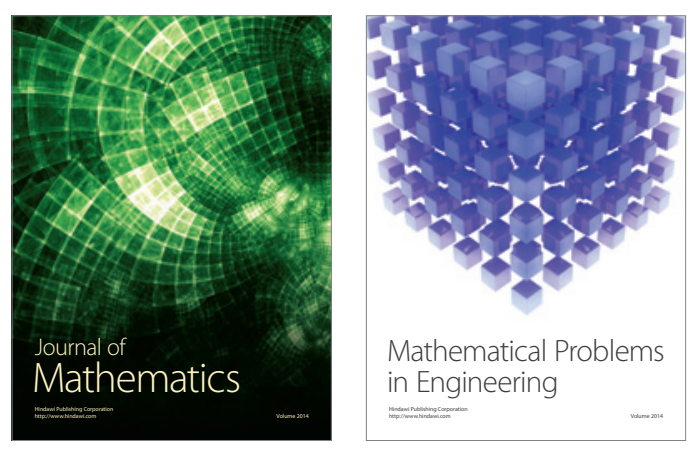

Mathematical Problems in Engineering
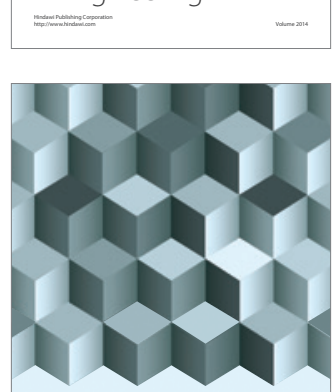

Journal of

Function Spaces
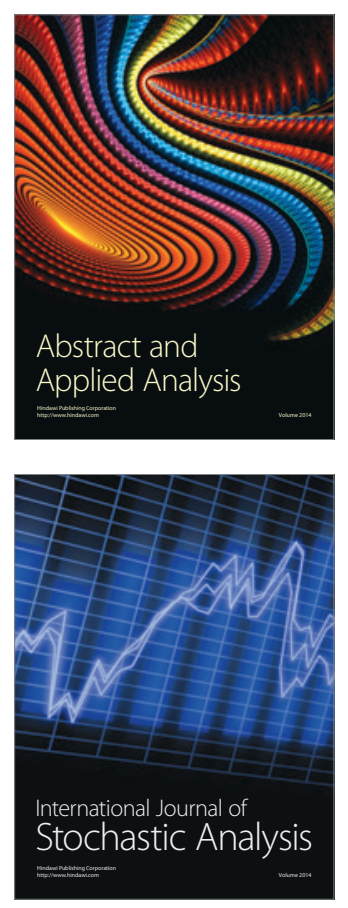

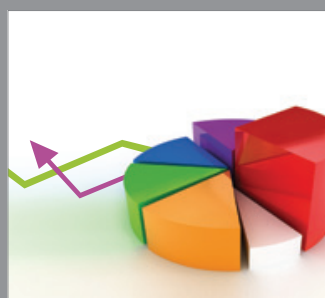

ournal of

Probability and Statistics

Promensencen
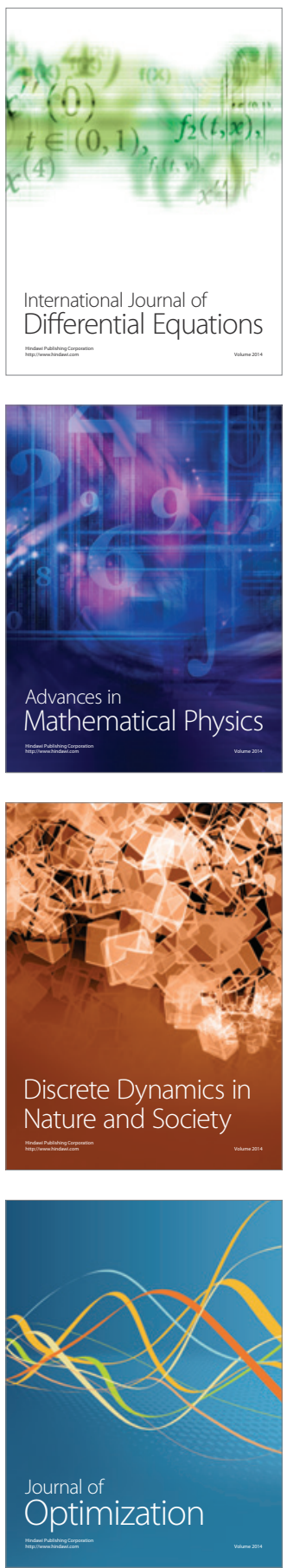